\title{
Dispersion-convolution model for simulating peaks in a flow injection system
}

\author{
Su-Cheng Pai ${ }^{a}{ }^{a}$, , Yee-Hwong Lai ${ }^{\text {a,b }}$, Ling-Yun Chiao ${ }^{c}$, Tiing Yu ${ }^{d}$ \\ a Division of Marine Chemistry, Institute of Oceanography, National Taiwan University, Taipei, Taiwan \\ ${ }^{\mathrm{b}}$ Chemical Laboratory, National Center of Ocean Research, Taipei, Taiwan \\ ${ }^{\mathrm{c}}$ Division of Marine Geology and Geophysics, Institute of Oceanography, National Taiwan University, Taipei, Taiwan \\ ${ }^{\mathrm{d}}$ Department of Applied Chemistry, National Chiao Tung University, Hsinchu, Taiwan \\ Received 12 April 2006; received in revised form 28 October 2006; accepted 3 November 2006 \\ Available online 20 November 2006
}

\begin{abstract}
A dispersion-convolution model is proposed for simulating peak shapes in a single-line flow injection system. It is based on the assumption that an injected sample plug is expanded due to a "bulk" dispersion mechanism along the length coordinate, and that after traveling over a distance or a period of time, the sample zone will develop into a Gaussian-like distribution. This spatial pattern is further transformed to a temporal coordinate by a convolution process, and finally a temporal peak image is generated. The feasibility of the proposed model has been examined by experiments with various coil lengths, sample sizes and pumping rates. An empirical dispersion coefficient $\left(D^{*}\right)$ can be estimated by using the observed peak position, height and area $\left(t_{\mathrm{p}}^{*}, h^{*}\right.$ and $\left.A_{\mathrm{t}}^{*}\right)$ from a recorder. An empirical temporal shift $\left(\Phi^{*}\right)$ can be further approximated by $\Phi^{*}=D^{*} / u^{2}$, which becomes an important parameter in the restoration of experimental peaks. Also, the dispersion coefficient can be expressed as a second-order polynomial function of the pumping rate $Q$, for which $D^{*}(Q)=\delta_{0}+\delta_{1} Q+\delta_{2} Q^{2}$. The optimal dispersion occurs at a pumping rate of $Q_{\text {opt }}=\sqrt{\delta_{0} / \delta_{2}}$. This explains the interesting "Nike-swoosh" relationship between the peak height and pumping rate. The excellent coherence of theoretical and experimental peak shapes confirms that the temporal distortion effect is the dominating reason to explain the peak asymmetry in flow injection analysis.
\end{abstract}

(C) 2006 Elsevier B.V. All rights reserved.

Keywords: Dispersion-convolution model; Flow injection analysis; Peak simulation

\section{Introduction}

Flow injection analysis (FIA) was first introduced by Ruzick and Hansen in 1975 [1], and has been widely adopted as an efficient analytical tool in many scientific fields. Although the principle seems to be well understood, Kolev [2] has suggested that the theoretical foundation for generating a flow injection peak is still far from complete due to the complexity of mechanisms involved (dispersion, convection and other kinetic reasons). He concluded that, in general, the Uniform Dispersion Model (UDM) [3,4] and Random Walk Model (RWM) [5-7] are theoretically preferred. But, they require difficult mathematics and computations, and thus have limited utilization in a practi-

\footnotetext{
* Corresponding author at: Division of Marine Chemistry, Institute of Oceanography, National Taiwan University, P.O. Box 23-13, Taipei, Taiwan. Tel.: +886 2 23627358; fax: +886223632912.

E-mail address: scpai@ntu.edu.tw (S.-C. Pai).
}

cal system. On the other hand, the Tanks-in-Series Model (TSM) $[8,9]$ and Axially Dispersed Plug Flow Model (ADPFM) [10] have gained more popularity not only because of the lesser mathematics involved, but also due to the fact that it is not necessary to know the exact flow pattern in a tubular system.

Apart from those models, several mathematical approaches have also been proposed to construct a peak curve including the Exponentially-Modified Gaussian functions (EMG) $[11,12]$ and the Polynomial-Modified Gaussian functions (PMG) [13-17]. Recently, a Temporally-Convoluted Gaussian equation (TCG) [18] has been developed which is not only the simplest, but also indicates that a very basic and long-ignored principle can be an important key to solve the ambiguous skewed peak problems. This equation involves only two basic principles: (1) that the expansion of the sample zone is proportional to the square root of distance or time travelled; and (2) that the concentration profile is gradually turned into a Gaussian-like distribution along the tubular channel. The difference between this approach and 
all previous models is that it includes a temporal convolution process, in which all spatial parameters need to be convoluted along a temporal coordinate so as to simulate the peak that is shown on the recorder. Later, Pai and Chiao [19] have suggested that a "shift", denoted as $\Phi$, should exist universally between an apparent peak position $t_{\mathrm{p}}^{*}$ and its actual arrival time $t_{\mathrm{p}}$ $\left(\Phi=t_{\mathrm{p}}-t_{\mathrm{p}}^{*}\right)$ for any flow-type instrument with a single fixed detector. When restoring an experimental peak, this $\Phi$ should be added to modify the peak position term of a Gaussian equation. Also, the same $\Phi$ becomes a parameter to describe the expanding nature of the flow system, for which $\Phi \approx D / u^{2}$ where $D$ is a longitudinal dispersion coefficient and $u$ the flow speed. Those findings have been combined, re-assessed and extended in this work, and a new model is proposed here and has been thoroughly examined and verified by experiments using a self-made single-line flow injection system. The relationship between the dispersion coefficient $D$ and flow speed $u$ (or pumping rate $Q$ ) will also be studied both theoretically and experimentally.

\section{Theoretical}

\subsection{Parameters in a flow injection system}

A typical single-line flow injection system is illustrated in Fig. 1 (i). The basic tubular channel consists of an injector and a detector with a mixing coil in between. The corresponding internal volumes are $V_{\mathrm{i}}, V_{\mathrm{d}}$ and $V_{\mathrm{c}}$, respectively. When summing up the total tubular volume $\left(V_{\text {total }}\right)$, only half of the injector and detector volumes $\left(V_{\mathrm{i}}\right.$ and $\left.V_{\mathrm{d}}\right)$ are taken into account, i.e. $V_{\text {total }}$ (unit: $\mathrm{ml})=0.5 V_{\mathrm{i}}+V_{\mathrm{c}}+0.5 V_{\mathrm{d}}$ [1]. Since the liquid is pumped at a consistent rate $Q\left(\mathrm{ml} \mathrm{s}^{-1}\right)$, and the tubing is assumed to have a uniform cross-section area $a\left(\mathrm{~cm}^{2}\right)$, the average migration speed of the sample can be calculated as $u\left(\mathrm{~cm} \mathrm{~s}^{-1}\right)=Q / a$. The total channel length from the center of the injector to the center of the detector is $L_{\text {total }}(\mathrm{cm})$, which is calculated by $L_{\text {total }}$ $(\mathrm{cm})=V_{\text {total }} / a$. The mean residence time for the mass center to arrive at the detector is $t_{\mathrm{p}}(\mathrm{s})=V_{\text {total }} / Q$ or $L_{\text {total }} / u$. A sample plug is characterized by an initial concentration $C_{0}(\mu \mathrm{M})$ and an injection volume $V_{\mathrm{i}}(\mathrm{ml})$. An equivalent initial zone width $W_{\mathrm{i}}(\mathrm{cm})$ can be obtained by $W_{\mathrm{i}}=V_{\mathrm{i}} / a$. This sample plug can be plotted on a "conc. versus length" diagram (Fig. 1(ii)), so that the area is $C_{0} W_{\mathrm{i}}(\mu \mathrm{M} \mathrm{cm})$. The area remains constant during the migration of the sample zone along the $L$ coordinate.

To cope with the reading of the detecting device, the above diagram needs to be further converted to a "signal versus length" plot (Fig. 1(iii)). Accordingly, the initial concentration $C_{0}$ becomes $S_{0}$ (signal units), where $S_{0}=\eta C_{0}$ and $\eta$ is the sensitivity (signal- $\mu \mathrm{M}^{-1}$ ) of the detector. The plug area, denoted as $A_{\mathrm{L}}$ (signal-cm), remains $S_{0} W_{\mathrm{i}}$, even though the sample zone has spread out after traveling over a distance.

\subsection{The Gaussian approximation}

In the present study, the expanding of the sample zone is assumed to be attributed to a "bulk" longitudinal dispersion mechanism [20], which results in a Gaussian distribution pattern after traveling a distance or a period of time. A Gaussian
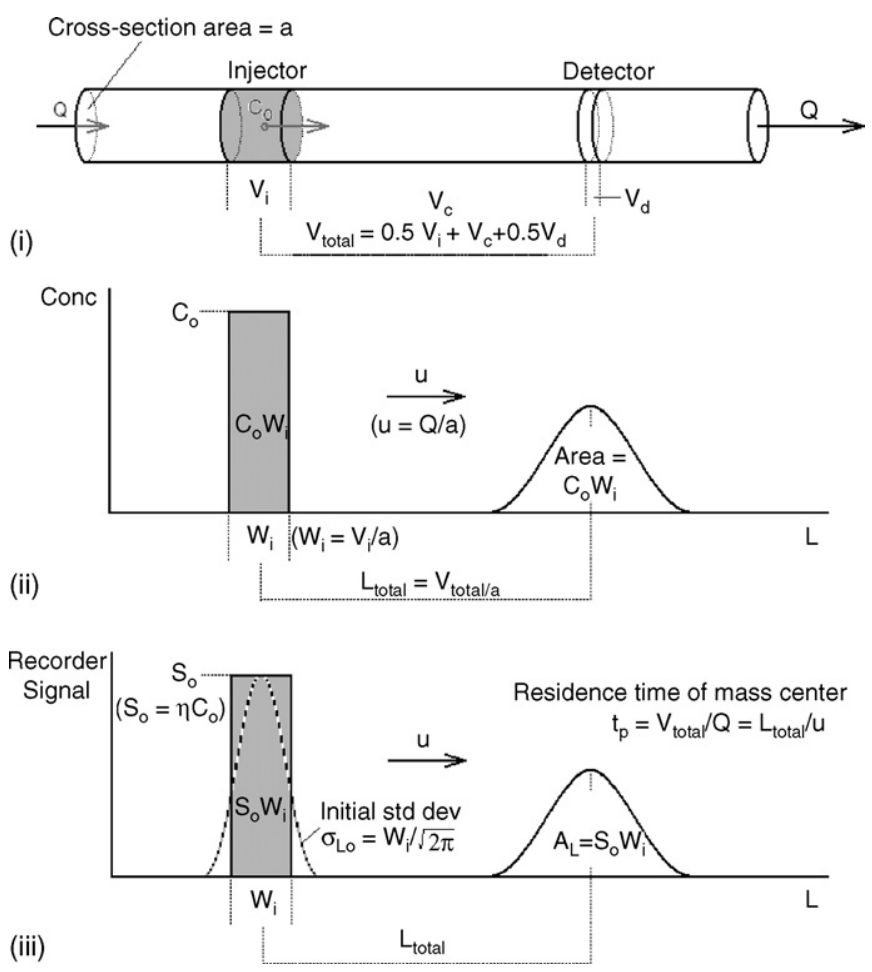

Fig. 1. Parameters involved in an ideal single-line flow injection system. (i) A sample plug with a concentration $C_{0}(\mu \mathrm{M})$ and volume $V_{\mathrm{i}}(\mathrm{ml})$ is injected into a tubular system, which has a cross-section of $a\left(\mathrm{~cm}^{2}\right)$ and the flow is pumped at a rate of $Q\left(\mathrm{ml} \mathrm{s}^{-1}\right)$. The total tubular volume $V_{\text {total }}(\mathrm{ml})$ is measured from the center of the injector to the center of the detector, $V_{\text {total }}=0.5 V_{\mathrm{i}}+V_{\mathrm{c}}+0.5 V_{\mathrm{d}}$. (ii) The same system is plotted on an $L$ coordinate, with an initial zone width $W_{\mathrm{i}}$ $(\mathrm{cm})=V_{\mathrm{i}} / a$, and an average flow speed $u\left(\mathrm{~cm} \mathrm{~s}^{-1}\right)=Q / a$. The plug area remains conservative during the migration $\left(\right.$ Area $\left.=C_{0} W_{\mathrm{i}}\right)$. (iii) The vertical coordinate is converted to the recorder signal $S$, so the initial plug height is $S_{0}=\eta C_{0}$, where $\eta$ (signal- $\left.\mu \mathrm{M}^{-1}\right)$ is the sensitivity of the recorder. The plug area $\left(A_{\mathrm{L}}\right.$, unit: signal-cm) becomes $A_{\mathrm{L}}=S_{0} W_{\mathrm{i}}$. The initial plug can be converted to a hypothetical Gaussian distribution pattern with an initial standard deviation $\sigma_{\mathrm{L} 0}(\mathrm{~cm})$ $=W_{\mathrm{i}} / \sqrt{2 \pi}$. The mean residence time for the mass center to arrive at the detector is $t_{\mathrm{p}}(\mathrm{s})=V_{\text {total }} / Q$ or $L_{\text {total }} / u$.

function comprises three major parameters: the peak area $A_{\mathrm{L}}$, the standard deviation $\sigma_{\mathrm{L}}$ at the detector, and the peak position $L_{\mathrm{p}}$. The latter two terms refer to a mean residence time $t_{\mathrm{p}}$ at a given flow speed $u$. Therefore, at the detector, the standard deviation is $\sigma_{\mathrm{L}}\left(t_{\mathrm{p}}\right)$ and the peak position $L_{\mathrm{p}}\left(t_{\mathrm{p}}\right)=u t_{\mathrm{p}}$. Accordingly, the Gaussian pattern can be expressed by the following equation:

$S(L)=\frac{A_{\mathrm{L}}}{\sqrt{2 \pi} \sigma_{\mathrm{L}}\left(t_{\mathrm{p}}\right)} \mathrm{e}^{-\left(L-u t_{\mathrm{p}}\right)^{2} /\left(2 \sigma_{\mathrm{L}}\left(t_{\mathrm{p}}\right)^{2}\right)}$

The standard deviation at any instance is proportional to the square root of time, $\sigma_{\mathrm{L}}(t) \propto \sqrt{t}$, and the expanding rate of the variance $\left(\mathrm{d} \sigma_{\mathrm{L}}(t)^{2} / \mathrm{d} t\right)$ is a constant:

$\frac{\mathrm{d} \sigma_{\mathrm{L}}(t)^{2}}{\mathrm{~d} t}=2 D$

where $D$ (having a unit of $\mathrm{cm}^{2} \mathrm{~s}^{-1}$ ) is defined as the "bulk" longitudinal dispersion-diffusion coefficient at a constant flow speed. It comprises two major fractions, i.e. $D_{\mathrm{d}}$ and $D_{\mathrm{m}}$; the former is "dispersion" coefficient in the longitudinal direction and the latter is the "molecular diffusion" coefficient. It should be 
noted here that once the liquid starts to flow, $D_{\mathrm{d}}$ is much larger than $D_{\mathrm{m}}$, therefore the symbol $D$ in Eq. (2) can be regarded exclusively as a longitudinal dispersion coefficient.Integration of the above equation gives:

$\sigma_{\mathrm{L}}(t)^{2}\left(\mathrm{~cm}^{2}\right)=\sigma_{\mathrm{L} 0}^{2}+2 D t$

where $\sigma_{\mathrm{L} 0}^{2}$ refers to an initial variance at $t=0$.

By combining Eqs. (1) and (3), the longitudinal peak pattern at a given time $t_{\mathrm{p}}$ can be written as:

$S(L)=\frac{A_{\mathrm{L}}}{\sqrt{2 \pi \sigma_{\mathrm{L} 0}^{2}+4 \pi D t_{\mathrm{p}}}} \mathrm{e}^{-\left(L-u t_{\mathrm{p}}\right)^{2} /\left(2 \sigma_{\mathrm{L} 0}{ }^{2}+4 D t_{\mathrm{p}}\right)}$

\subsection{The initial state}

Although a sample plug may be small, the initial zone standard deviation $\sigma_{\mathrm{L} 0}$ should not be treated as zero, otherwise the calculation of Eq. (4) will lead to infinity at $t=0$. To avoid this problem, the initial standard deviation is defined here to be:

$\sigma_{\mathrm{L} 0}(\mathrm{~cm})=\frac{W_{\mathrm{i}}}{\sqrt{2 \pi}}$

or $\sigma_{\mathrm{L} 0} \approx 0.399 W_{\mathrm{i}}$. With this definition, the initial peak height $h$ (at $t=0$ ) will be identical to the initial signal of the sample:

$h_{(t=0)}=\frac{A_{\mathrm{L}}}{\sqrt{2 \pi} \sigma_{\mathrm{L} 0}}=\frac{A_{\mathrm{L}}}{W_{\mathrm{i}}}=S_{0}$

Apart from this initial state, the relationship between the peak width and standard deviation at any other time is still $W_{\mathrm{L}}(t)=4 \sigma_{\mathrm{L}}(t)$.

\subsection{Convolution of a spatial pattern to a temporal image}

When a sample zone passes through a detector located at $L=L_{\mathrm{p}}$, the recorder will give out a peak on the temporal coordinate which is different in shape from the spatial distribution pattern. A convolution process is required to transform the spatial image $S(L)$ to a temporal function $S(t)$. The transformations of individual peak parameters $\left(L_{\mathrm{p}}\left(t_{\mathrm{p}}\right), A_{\mathrm{L}}\right.$ and $\left.\sigma_{\mathrm{L}}\left(t_{\mathrm{p}}\right)\right)$ from a longitudinal coordinate to a temporal axis $\left(t_{\mathrm{p}}, A_{\mathrm{t}}\right.$ and $\left.\sigma_{\mathrm{t}}\left(t_{\mathrm{p}}\right)\right)$ are described below:

$t_{\mathrm{p}}(\mathrm{s})=\frac{L_{\mathrm{p}}}{u}$

$A_{\mathrm{t}}($ signal-s $)=\frac{A_{\mathrm{L}}}{u}$

The standard deviation term is no longer fixed at a specific $t_{\mathrm{p}}$, but variable with time:

$\sigma_{\mathrm{t}}(t)(\mathrm{s})=\frac{\sigma_{\mathrm{L}}(t)}{u}$

The resultant temporally convoluted Gaussian equation (TCG) $[18,19]$ becomes:

$S(t)=\frac{A_{\mathrm{t}}}{\sqrt{2 \pi \sigma_{\mathrm{L} 0^{2}} / u^{2}+4 \pi D t / u^{2}}} \mathrm{e}^{-\left(t-t_{\mathrm{p}}\right)^{2} /\left(2 \sigma_{\left.\mathrm{L} 0^{2} / u^{2}+4 D t / u^{2}\right)}\right.}$
This function imitates the temporal peak as normally seen on a recorder. It is not symmetrical, and the peak position appears at $t_{\mathrm{p}}^{*}$, slightly earlier than the expected $t_{\mathrm{p}}$. The apparent peak height $h^{*}\left(\right.$ at $\left.t=t_{\mathrm{p}}^{*}\right)$ is slightly higher than the expected $h\left(\right.$ at $\left.t=t_{\mathrm{p}}\right)$.

A numerical test is illustrated in Fig. 2. Assuming that a flow injection system is composed of $0.8 \mathrm{~mm}$ ID tubing, and that the carrier flow is running at a rate of $Q=0.04 \mathrm{ml} \mathrm{s}^{-1}$. A sample plug of $V_{\mathrm{i}}=0.100 \mathrm{ml}$ is injected with an initial signal of $S_{0}=1.000$, and three hypothetical detectors are located at $L_{\text {total }}=200,400$ and $1000 \mathrm{~cm}$. A hypothetical dispersion coefficient $(D)$ is designated as $100 \mathrm{~cm}^{2} \mathrm{~s}^{-1}$. The sample zone arrives at the three detectors at $t_{\mathrm{p}}=L / u=L a / Q=25.14,50.27$ and $125.68 \mathrm{~s}$, respectively. The corresponding heights of the spatial peaks are $h=0.1112,0.0789$ and 0.0500 . When these peak patterns are to be transformed onto a temporal axis, the apparent peak positions $\left(t_{\mathrm{p}}^{*}\right)$ are $23.61,48.72$ and $124.11 \mathrm{~s}$. The temporal shifts are $1.53,1.55$ and $1.57 \mathrm{~s}$, respectively. The apparent peak heights $\left(h^{*}\right)$ are $0.1130,0.0795$ and 0.0502 , and all are slightly higher than the corresponding $h$.

\subsection{The temporal shift}

The temporal shift (represents the shift of the peak position from an expected $t_{\mathrm{p}}$ to an apparent $t_{\mathrm{p}}^{*}$, i.e. $\Phi(\mathrm{s})=\left(t_{\mathrm{p}}-t_{\mathrm{p}}^{*}\right)$ is a quite consistent value for a fixed flow system when $t_{\mathrm{p}}$ is not too small. Its scale is related to both the dispersion coefficient $D$ and flow speed $u$. An approximate relationship has been derived as [19]:

$\Phi(\mathrm{s}) \approx \frac{D}{u^{2}}$

Thus, $\Phi$ can be calculated from $D$, but the latter may vary from system to system so it needs to be estimated experimentally.

\subsection{The dispersion coefficient $D$ of a given system}

Each flow injection system will have a specific dispersion coefficient $D$. However, it is almost impossible to obtain the $D$ value directly from a "spatial" peak pattern because such a pattern is not available when using a single fixed detector. Practically, the $D$ value could be estimated from "temporal" peak parameters, but a "recursion" process is necessary.

According to Eq. (10), the temporal peak height $h^{*}$ should appear at $t=t_{\mathrm{p}}^{*}$ :

$h^{*}=S\left(t_{\mathrm{p}}^{*}\right) \approx \frac{A_{\mathrm{t}}}{\sqrt{2 \pi \sigma_{\mathrm{L} 0}^{2} / u^{2}+4 \pi D t_{\mathrm{p}}^{*} / u^{2}}} Z$

where $Z$ is the exponential part of the Gaussian function:

$Z=\mathrm{e}^{-\left(t_{\mathrm{P}}^{*}-t_{\mathrm{p}}\right)^{2} /\left(2 \sigma_{\mathrm{L} 0}{ }^{2} / u^{2}+4 D t_{\mathrm{P}}^{*} / u^{2}\right)}$

It would be difficult to solve $D$ directly from Eq. (12). However, one may first assume the temporal peak height $h^{*}$ is nearly equal 

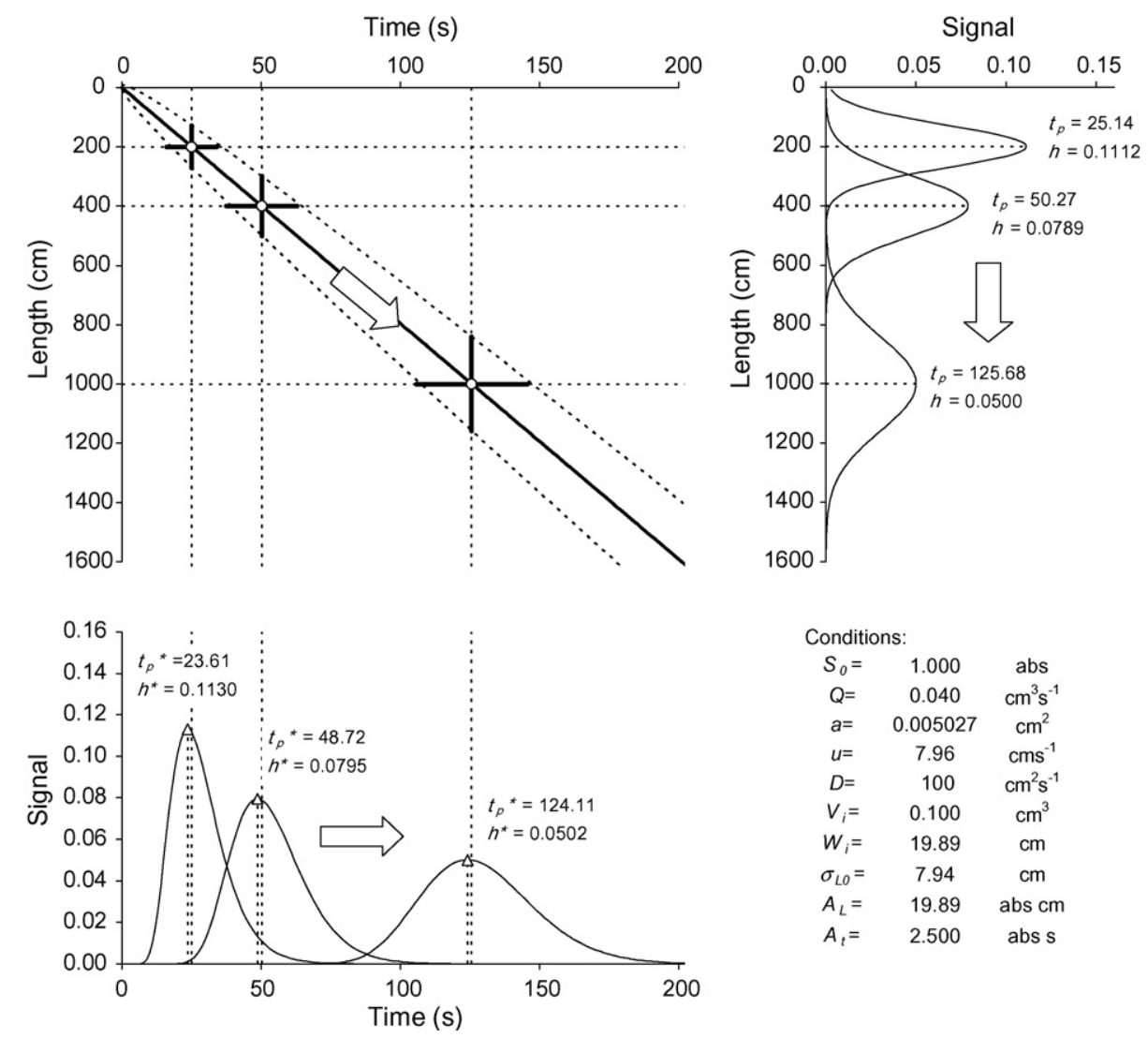

Fig. 2. Diagrams illustrating the evolution of a sample plug on a length vs. time diagram, at given conditions. The upper left diagram showing the migration route of the sample plug with two threshold boundaries $\left( \pm \sigma_{\mathrm{L}}(t)\right.$ ). Three detectors are located at $L_{\text {total }}=200,400$ and $1000 \mathrm{~cm}$, respectively. The mass distributions (spatial peaks) of the sample arriving at the detectors are shown in the right diagram, whereas the temporal peaks are plotted in the bottom diagram. Circles denote the true peak positions $\left(t_{\mathrm{p}}\right)$; triangles represent the observed peak positions $\left(t_{\mathrm{p}}^{*}\right)$; the latter are always slightly earlier than that expected by a nearly-consistent temporal shift, with apparent heights $\left(h^{*}\right)$ slightly higher than that of spatial peaks $(h)$.

to the spatial peak height $h$. In other words, one may first let $Z=1$ :

$h^{*} \approx S\left(t_{\mathrm{p}}^{*}\right) \approx \frac{A_{t}}{\sqrt{2 \pi \sigma_{\mathrm{L} 0}^{2} / u^{2}+4 \pi D t_{\mathrm{p}}^{*} / u^{2}}}$

A first estimation of $D$ can be obtained:

$D(1$ st est. $)=\frac{A_{\mathrm{t}}^{2} u^{2}}{4 \pi h^{* 2} t_{\mathrm{p}}^{*}}-\frac{\sigma_{\mathrm{L} 0}^{2}}{2 t_{\mathrm{p}}^{*}}$

By putting this first approximated $D$ into Eq. (13) and letting $t_{\mathrm{p}}-t_{\mathrm{p}}^{*}=\Phi \approx D / u^{2}$, a $Z$ value is obtained. This $Z$ value is put back into Eq. (12) and a second estimation of $D$ is given:

$D(2$ nd est. $)=\frac{A_{\mathrm{t}}^{2} u^{2} Z^{2}}{4 \pi h^{* 2} t_{\mathrm{p}}^{*}}-\frac{\sigma_{\mathrm{L} 0}^{2}}{2 t_{\mathrm{p}}^{*}}$

A numerical test is demonstrated in Table 1 using the data provided in Fig. 2. In that system, the dispersion coefficient $D$ has been designated as $100 \mathrm{~cm}^{2} \mathrm{~s}^{-1}$ and other conditions $\left(A_{\mathrm{t}}, u, \sigma_{\mathrm{L} 0, \ldots}\right)$ are fixed. At each given observation point (e.g. $L=200,400$ or $1000 \mathrm{~cm}$ ), a temporal peak is generated, and three peak parameters $\left(A_{\mathrm{t}}^{*}, t_{\mathrm{p}}^{*}\right.$ and $\left.h^{*}\right)$ are obtained from that peak. By putting these data into the above equations, a first estimation of $D$ by Eq. (15) gives the values of 103.12, 101.62 and

Table 1

Numerical test showing the fesibility of estimating the dispersion coefficient $D$ values from temporal peak parameters by a recursion calculation method (the $D$ value is designated to be $100 \mathrm{~cm}^{2} \mathrm{~s}^{-1}$ )

\begin{tabular}{|c|c|c|c|c|c|c|c|c|}
\hline \multirow{2}{*}{$\begin{array}{l}\text { Channel length } \\
L_{\text {total }}(\mathrm{cm})\end{array}$} & \multicolumn{2}{|l|}{ Spatial peak } & \multicolumn{2}{|l|}{ Temporal peak } & \multirow{2}{*}{$\begin{array}{l}\text { Temporal } \\
\text { shift } \Phi(\mathrm{s})\end{array}$} & \multicolumn{3}{|c|}{ Estimation of $D$ and $\Phi$ values } \\
\hline & Position $t_{\mathrm{p}}(\mathrm{s})$ & Height $h$ (abs) & Position $t_{\mathrm{p}}^{*}(\mathrm{~s})$ & Height $h^{*}$ (abs) & & $\begin{array}{l}D \text { (1st est.) } \\
\left(\mathrm{cm}^{2} \mathrm{~s}^{-1}\right)\end{array}$ & $\begin{array}{l}D \text { (2nd est.) } \\
\left(\mathrm{cm}^{2} \mathrm{~s}^{-1}\right)\end{array}$ & $\Phi$ (est.) (s) \\
\hline 400 & 50.27 & 0.0789 & 48.72 & 0.0795 & 1.55 & 101.62 & 99.96 & 1.58 \\
\hline 1000 & 125.68 & 0.0500 & 124.11 & 0.0502 & 1.57 & 100.43 & 99.79 & 1.58 \\
\hline
\end{tabular}

Both spatial and temporal peak data are generated by Eqs. (4) and (10). Conditions refer to Fig. 2. The iterating estimation of the $D$ value follows Eqs. (15) and (16). The estimated temporal shift is calculated by $\Phi$ (est.) $=D(2$ nd est. $) / u^{2}$. 
$100.43 \mathrm{~cm}^{2} \mathrm{~s}^{-1}$, respectively, with errors up to $3.12 \%$. Through a second estimation, the $D$ values are more accurate (99.62, 99.96 and $99.79 \mathrm{~cm}^{2} \mathrm{~s}^{-1}$ ), and the errors are effectively reduced to less than $0.4 \%$. Although more iterations can be done, the results from the second estimation are deemed close enough to the original value of $D=100 \mathrm{~cm}^{2} \mathrm{~s}^{-1}$. In this case, a temporal shift $(\Phi)$ of ca. $1.58 \mathrm{~s}$ can be estimated for this system, which is close to the theoretical values of $1.53-1.57 \mathrm{~s}$. This numerical test demonstrates that the $D$ value of a system can indeed be retrieved from experimental peak parameters.

\section{Experimental}

\subsection{Instrument layout}

A flow injection system was assembled as illustrated in Fig. 3. It consisted of (i) a speed-adjustable peristaltic pump; (ii) a Rheodyne six-port injector with a changeable sample loop to give a specific injection size; (iii) a changeable mixing coil made by winding a desired length of Teflon tube $(0.8 \mathrm{~mm}$ ID) onto a $1 \mathrm{~cm}$ diameter supporting rod; (iv) a Shimadzu 160A double beam spectrophotometer installed with a Hellma flow cuvette $(1 \mathrm{~cm}$ light path with a capacity of $31 \mu \mathrm{l})$.

The spectrophotometer was operated in the "time-scan" mode, so absorbance readings $S_{\exp }(t)$ were recorded sequentially after the sample injection at required intervals $(\Delta t=0.1-5 \mathrm{~s})$.

\subsection{Dye solution}

A food dye "blue \#1" solution (maximum absorptivity at $629 \mathrm{~nm}$ ) was prepared for the injection test. The absorbance of a test sample when filled up into the flow cuvette was regarded as the initial signal $S_{0}$.

\subsection{Volumetric measurement}

The actual sample volume injected $\left(V_{\mathrm{i}}\right)$ was identified by injecting a dense dye solution into the injector and collecting the effluent in a $100 \mathrm{ml}$ graduated flask and diluting to the mark. The volume was calculated by comparing the absorbance that was obtained by adding a known volume of the same dye solution and

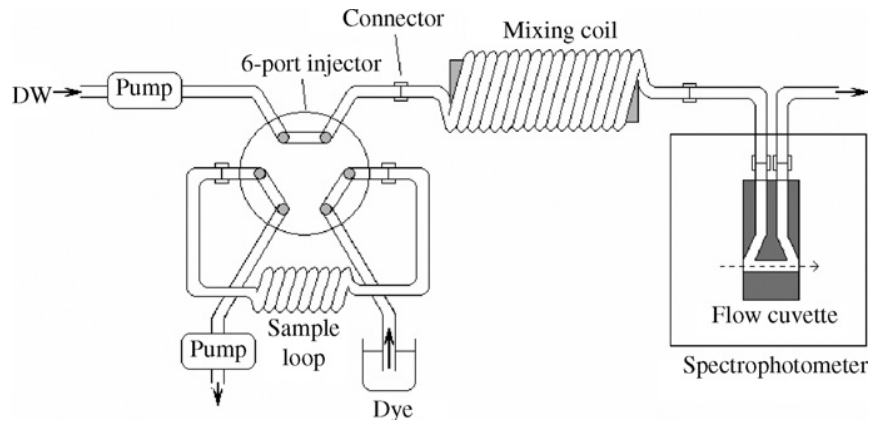

Fig. 3. The flow injection device used in this study consists of a peristaltic pump, a 6-port injector with a sample loop, a mixing coil of designated length, and a Hellma flow cuvette $(1 \mathrm{~cm}, 31 \mu \mathrm{l})$ installed in a Shimadzu 160A spectrophotometer. diluting to the same volume. In this way, $V_{\mathrm{i}}$ could be measured to a precision of better than $\pm 2 \mu \mathrm{l}$. The volume of the flow channel ( $V_{\mathrm{c}}$, including the coil and connectors) and the detector $\left(V_{\mathrm{d}}\right)$ was also estimated in a similar way by filling with a dye solution and then draining it out for quantification. The pumping rate $(Q)$ was measured just prior to each experiment by weighing the outflow water in a dry beaker over a suitable time span.

\subsection{Calculation of channel length}

A series of coils were made of $0.8 \mathrm{~mm}$ ID Teflon tube, which gave a cross section area of $a=0.005027 \mathrm{~cm}^{2}$. The length of each coil was decided not by a ruler, but calculated from the volumetric measurement. For example, the capacity of a coil was measured to be $V_{\mathrm{c}}=2.12 \mathrm{ml}$, and then its length was calculated to be $L_{\mathrm{c}}=V_{\mathrm{c}} / a=422 \mathrm{~cm}$. The half of the cuvette volume plus connecting tubing was estimated to be ca. $0.18 \mathrm{ml}$, which was equivalent to $36 \mathrm{~cm}$ in length. The combined length was $422+36=458 \mathrm{~cm}$. When an injection volume of $V_{\mathrm{i}}=0.112 \mathrm{ml}$ was applied, the initial width was $W_{\mathrm{i}}=22.28 \mathrm{~cm}$. An extra length of $0.5 W_{\mathrm{i}}=11.14 \mathrm{~cm}$ should be added to compensate for the initial shift of the mass center. In this way, the total channel length $\left(L_{\text {total }}\right)$ of this case was calculated to be ca. $469 \mathrm{~cm}$.

\subsection{Measurements of peak parameters}

The injection of a sample and the switching-on of the recorder were controlled simultaneously. Each injection action produced a temporal peak on the recorder, which provided a temporal peak position $t_{\mathrm{p}}^{*}$ and an apparent peak height $h^{*}$. The experimental temporal peak area (denoted as $A_{\mathrm{t}}^{*}$ ) was estimated by summing up all absorbance values $S(t)$ and multiplying the recording interval $\Delta t$, i.e. $A_{\mathrm{t}}^{*}=\Sigma S(t) \times \Delta t$.

\subsection{Calculation of the empirical $D^{*}$}

In Section 2.6, the dispersion coefficient can be theoretically estimated by Eqs. (12)-(16), but experimentally the flow speed $u$ and initial standard deviation $\sigma_{\mathrm{L} 0}$ still can not be directly measured. Also, the flow injection device may involve some physical effects other than the longitudinal dispersion alone. For practical reasons, the pumping rate $Q$ and injection volume $V_{\mathrm{i}}$ are used instead of $u$ and $\sigma_{\mathrm{L} 0}$, and the experimentally retrieved $D$ value in the following sections is named the "empirical" dispersion coefficient and denoted as $D^{*}$.

Since $u=Q / a, \sigma_{\mathrm{L} 0}=W_{\mathrm{i}} / \sqrt{2 \pi}$ and $W_{\mathrm{i}}=V_{\mathrm{i}} / a$, the first approximation of $D$ by Eq. (15) is rewritten as:

$D^{*}(1$ st est. $)=\frac{A_{\mathrm{t}}^{* 2} Q^{2}-V_{\mathrm{i}}^{2} h^{* 2}}{4 \pi a^{2} h^{* 2} t_{\mathrm{p}}^{*}}$

The temporal shift estimated from experimental data should also be empirical and is denoted as $\Phi^{*} \approx D^{*} a^{2} / Q^{2}$. Similarly, Eq. (13) becomes:

$Z^{*}=\mathrm{e}^{-\left(D^{* 2} a^{4} \pi\right) /\left(Q^{2}\left(V_{\mathrm{i}}^{2}+4 \pi D^{*} a^{2} t_{\mathrm{p}}^{*}\right)\right.}$ 
By putting $Z^{*}$ back into consideration, a second estimation is obtained by:

$D^{*}(2$ nd est. $)=\frac{A_{\mathrm{t}}^{* 2} Q^{2} Z^{* 2}-V_{\mathrm{i}}^{2} h^{* 2}}{4 \pi a^{2} h^{* 2} t_{\mathrm{p}}^{*}}$

The unit for $D^{*}$ is $\mathrm{cm}^{2} \mathrm{~s}^{-1}$.

\subsection{Restoration of experimental peaks}

To restore an experimental peak, it is necessary to compose three peak parameters, i.e. the temporal peak area $\left(A_{\mathrm{t}}^{*}\right)$, the peak position $t_{\mathrm{p}}$ and the standard deviation $\left(\sigma_{\mathrm{t}}(t)\right)$ into the TCG equation (Eq. (10)). The peak position $\left(t_{\mathrm{p}}\right)$ can not be read from the recorder but can be estimated by adding a shift $\Phi^{*}$ to the apparent peak position $t_{\mathrm{P}}^{*}$, i.e. $t_{\mathrm{p}}=t_{\mathrm{p}}^{*}+\Phi^{*}$.

The longitudinal standard deviation is:

$\sigma_{\mathrm{L}}(t)(\mathrm{cm})=\sqrt{\sigma_{\mathrm{L} 0}^{2}+2 D^{*} a^{2} t / Q^{2}}$

which needs to be converted to a temporal standard deviation:

$\sigma_{\mathrm{t}}(t)(\mathrm{s})=\frac{\sigma_{\mathrm{L}}(t)}{u}=\sqrt{\frac{\sigma_{\mathrm{L} 0}^{2}+2 D^{*} t}{u^{2}}}$

Accordingly, a restored peak function $S^{\prime}(t)$ is expressed as:

$$
\begin{aligned}
S^{\prime}(t)= & \frac{A_{\mathrm{t}}^{*}}{\sqrt{2 \pi \sigma_{\mathrm{L} 0}^{2} / u^{2}+4 \pi D^{*} t / u^{2}}} \\
& \times \mathrm{e}^{-\left(t-t_{\mathrm{p}}^{*}-D^{*} / u^{2}\right)^{2} /\left(2 \sigma_{\mathrm{L} 0}^{2} / u^{2}+4 D^{*} t / u^{2}\right)}
\end{aligned}
$$

Then, $\sigma_{\mathrm{L} 0}$ and $u$ are replaced by $V_{\mathrm{i}}$ and $Q\left(\sigma_{\mathrm{L} 0}^{2}=V_{\mathrm{i}}^{2} /\left(2 \pi a^{2}\right)\right)$; $u=Q / a)$ :

$S^{\prime}(t)=\frac{A_{\mathrm{t}}^{*} Q}{\sqrt{V_{\mathrm{i}}^{2}+4 \pi a^{2} D^{*} t}} \mathrm{e}^{-\left(t-t_{\mathrm{p}}^{*}-D^{*} a^{2} / Q^{2}\right)^{2} \pi Q^{2} /\left(V_{\mathrm{i}}^{2}+4 \pi a^{2} D^{*} t\right)}$

Furthermore, since $\Phi^{*} \approx D^{*} a^{2} / Q^{2}$, it can also be written as:

$S^{\prime}(t)=\frac{A_{\mathrm{t}}^{*}}{\sqrt{V_{\mathrm{i}}^{2} / Q^{2}+4 \pi \Phi^{*} t}} \mathrm{e}^{-\left(t-t_{\mathrm{p}}^{*}-\Phi *\right)^{2} /\left(V_{\mathrm{i}}^{2} /\left(\pi Q^{2}\right)+4 \Phi t\right)}$

The peaks that restored by either Eq. (23) or Eq. (24) are identical.

\subsection{The residue curve}

The difference between a restored peak $S^{\prime}(t)$ and the original experimental peak $S_{\exp }(t)$ is in a crooked shape, which has been named here the residue curve:

$\xi(t)=S^{\prime}(t)-S_{\exp }(t)$

The amplitude of this residue curve (maximum to minimum) indicates the offset from the theoretical simulation quantitatively, which may include the influences of some physical effects other than the longitudinal dispersion.

\section{Results and discussion}

\subsection{Peak reproducibility}

The reproducibility of peaks generated by the flow injection system was quite good judging visually by overlapping replicated peaks on one diagram. Moreover, it was evaluated quantitatively by measuring the variations of the peak position, height and area (i.e. $t_{\mathrm{p}}^{*}, h^{*}$ and $A_{\mathrm{t}}^{*}$ ). A test was made for the system using an injection volume $\left(V_{\mathrm{i}}\right)$ of $0.499 \mathrm{ml}$ and a mixing coil length $\left(L_{\mathrm{c}}\right)$ of $328 \mathrm{~cm}$, running at a pumping rate $(Q)$ of $0.024 \mathrm{ml} \mathrm{s}^{-1}$. The average peak appearance time was: $t_{\mathrm{p}}^{*}=63.4 \pm 0.3 \mathrm{~s}(n=7)$; the average peak height was: $h^{*}=0.394 \pm 0.003(n=7)$; and the average peak area was: $A_{\mathrm{t}}^{*}=$ $12.43 \pm 0.08(n=7)$. All showed a relative precision (RSD) of better than $\pm 1 \%$. However, it was noticed that when the coil length was too short $\left(L_{\mathrm{c}}<70 \mathrm{~cm}\right)$, or the pumping rate was too fast $\left(Q>0.3 \mathrm{ml} \mathrm{s}^{-1}\right)$, erratic peak shapes would occur. The reproducibility was less satisfactory in those extreme conditions.

\subsection{Effect of coil length}

According to Ruzick and Hansen's textbook [1], changing the coil length from short to long will cause a longer peak appearance time and lower peak height, but the peak area will be conserved. These were proved experimentally and the data are shown in Table 2 . In this experiment, a series of coils $\left(L_{\mathrm{c}}=70\right.$, $112,215,328$ and $422 \mathrm{~cm}$ ) were used. The flow system was running at a constant pumping rate of $Q=0.016 \mathrm{ml} \mathrm{s}^{-1}$. The injection volume for all tests was $0.112 \mathrm{ml}$. A dye solution (absorbance $S_{0}=2.4$ ) was used for the injection.

In Fig. 4(A), the delay of the peak position was proportional to the increase of coil length $\left(t_{\mathrm{p}}^{*}=33.3,48.5,80.0,113.5\right.$ and $144.3 \mathrm{~s}$, respectively). The peak height dropped exponentially, i.e. $h^{*}=0.556,0.458,0.366,0.318$ and 0.299 , respectively. The peak areas were almost equal at $15.6 \pm 0.2$ for all tested coil lengths.

Calculation of the empirical dispersion coefficient $\left(D^{*}\right)$ was made by Eqs. (17)-(19) using the above data, to be between 15.2 and $18.4 \mathrm{~cm}^{2} \mathrm{~s}^{-1}$. A slightly decreasing trend was found for $D^{*}$ as the coil length increased. Although this phenomenon was not expected, it was understood that the system in use was not perfect. Thus, a larger dispersion might occur in the irregular space in the injector and detector, but this defect would have been reduced as the coil length increased. The temporal shifts were estimated by $\Phi^{*}=D^{*} a^{2} / Q^{2}$, to be in the range of $1.50-1.90 \mathrm{~s}$.

\subsection{Effect of injection volume}

An experiment was done by changing the sample loop with 7 sizes (total injection volume ranged from 0.046 to $0.573 \mathrm{ml}$, see Table 2) and the resultant peak shapes were recorded. The coil length used in this experiment was $328 \mathrm{~cm}$, and the system was running at a pumping rate of $Q=0.016 \mathrm{ml} \mathrm{s}^{-1}$. A dye solution with an absorbance of $S_{0}=0.89$ was used for the injection. In Fig. 4(B) it can be seen that the peak appearance time for the smallest volume $\left(V_{\mathrm{i}}=0.046 \mathrm{ml}\right)$ was found at $111.3 \mathrm{~s}$ and gradu- 
Table 2

Experimental conditions and resultant peak data

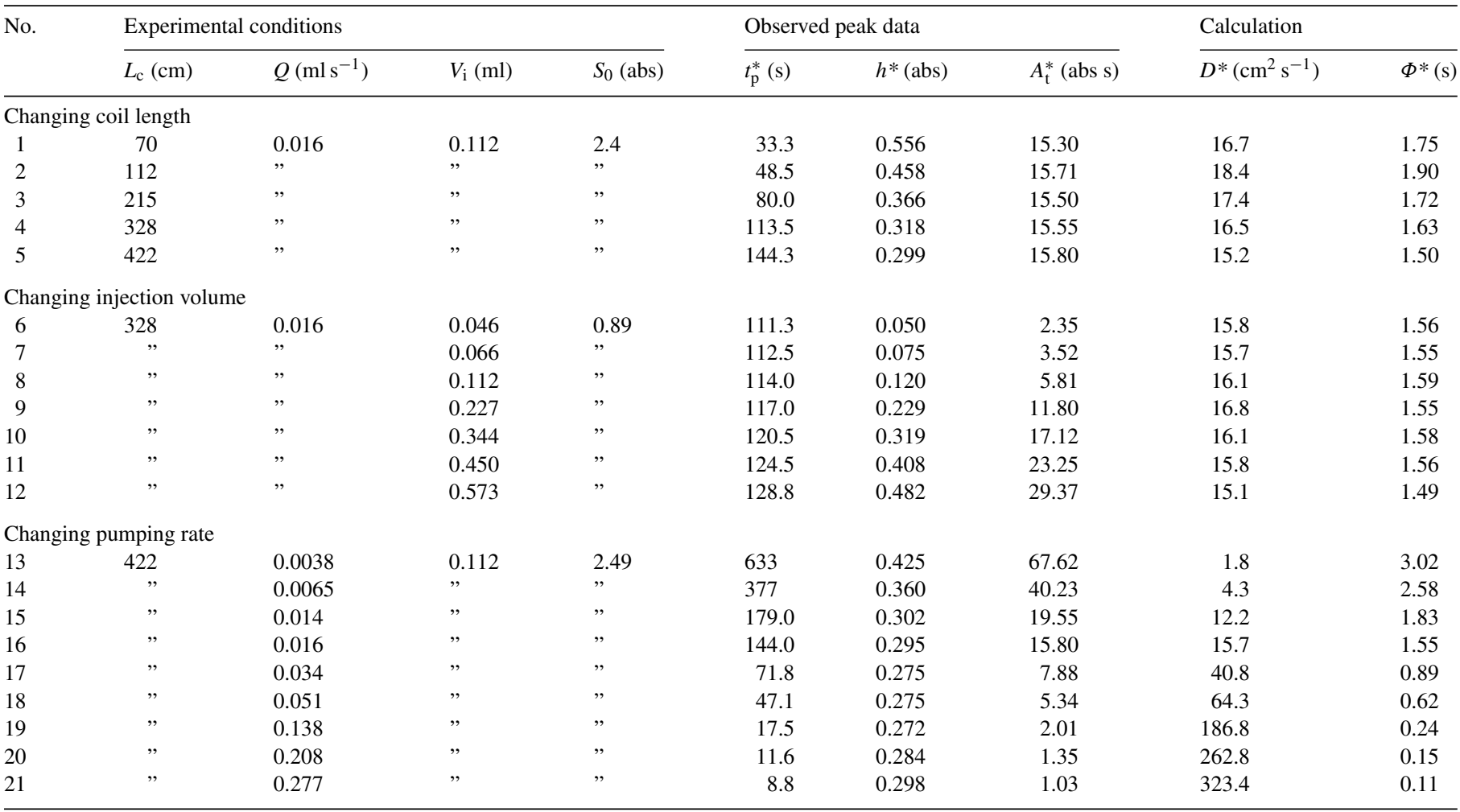

ally delayed to $128.8 \mathrm{~s}$ for the largest injection size of $0.573 \mathrm{ml}$. The peak heights increased exponentially, and the peak areas were increased proportionally with the injection volume, as they should be. The results of the $D^{*}$ calculation are listed in Table 2 and plotted in Fig. 4(B). All values were found almost equal at $15.9 \pm 0.5 \mathrm{~cm}^{2} \mathrm{~s}^{-1}$. The temporal shifts $\left(\Phi^{*}\right)$ in this case were then estimated to be ca. $1.56 \mathrm{~s}$.

\subsection{Effect of pumping rate}

The experimental conditions were: $V_{\mathrm{i}}=0.112 \mathrm{ml}$, $L_{\mathrm{c}}=422 \mathrm{~cm}$. A dye with an absorbance of ca. 2.49 was used. The pumping rate was adjusted from $Q=0.0038$ to $0.277 \mathrm{ml} \mathrm{s}^{-1}$. Experimental data are listed in Table 2, and were plotted against the pumping rate $Q$ (Fig. 5(A)) and also, its reciprocal $1 / Q$ (Fig. 5(B)). The peak appearance time $t_{\mathrm{p}}^{*}$ was found to be in a parabolic relationship with the pumping rate $Q$. This was confirmed by the linear line when the same data were plotted against $1 / Q$. Similar relationship was also found between the peak area $A_{\mathrm{t}}^{*}$ and $Q$.

The most interesting part was the effect of changing the pumping rate to the peak height $h^{*}$. At the slowest pumping rate $\left(Q=0.0038 \mathrm{ml} \mathrm{s}^{-1}\right), h^{*}$ was 0.425 . When the pumping rate was increased to $0.034-0.138 \mathrm{ml} \mathrm{s}^{-1}$, the peak height was decreased to $0.272-0.275$, but then increased to 0.298 at the fastest pumping rate of $0.277 \mathrm{ml} \mathrm{s}^{-1}$. The $h^{*}(Q)$ curve looks quite like a "Nike" shape (the "swoosh"), with a minimum at around $Q=0.1 \mathrm{ml} \mathrm{s}^{-1}$. A similar relationship can also be found on the $h^{*}$ versus $1 / Q$ diagram.
On the $D^{*}$ versus $Q$ diagram, the relationship is even more interesting. The correlation curve is not a linear one but shows an " $\mathrm{S}$ " shape (a slight turning at the bottom and top ends of the curve). When the pumping rate was minimal, $D^{*}$ approached nearly zero. This is reasonable because in a static situation only the molecular diffusion should be taken into account, which is at the level of $10^{-5} \mathrm{~cm}^{2} \mathrm{~s}^{-1}$. This relationship will be further discussed in a latter section (Section 4.6).

The temporal shift calculated could be as large as $3 \mathrm{~s}$ when the system was operating at the slowest pumping rate of $0.0038 \mathrm{ml} \mathrm{s}^{-1}$, but rapidly dropped to almost nothing when the pumping rate was faster than $0.138 \mathrm{ml} \mathrm{s}^{-1}$.

\subsection{Restoration of experimental peaks}

Each of the above experimental peaks provide three basic peak parameters (i.e. $t_{\mathrm{p}}^{*}, A_{\mathrm{t}}^{*}$ and $h^{*}$ ) and two calculated terms (i.e. the empirical dispersion coefficient $D^{*}$ and temporal shift $\Phi^{*}$ ). With this information, an experimental peak can be restored by Eq. (24). A total of 21 peaks were generated in this way and displayed in Figs. 6 and 7. Each peak was numbered according to the experimental conditions listed in Table 2 . The original recorder track (the experimental peak) was overlapped for comparison. The residue curve for each peak pair was also plotted.

In general, all peaks fit amazingly well if judged by the peak positions and heights, as well as the trends of asymmetry. However, small differences at the root of both sides of each peak can be identified on the diagrams. For most cases, the amplitude of the residue curve (normalized as \% of the peak height) 

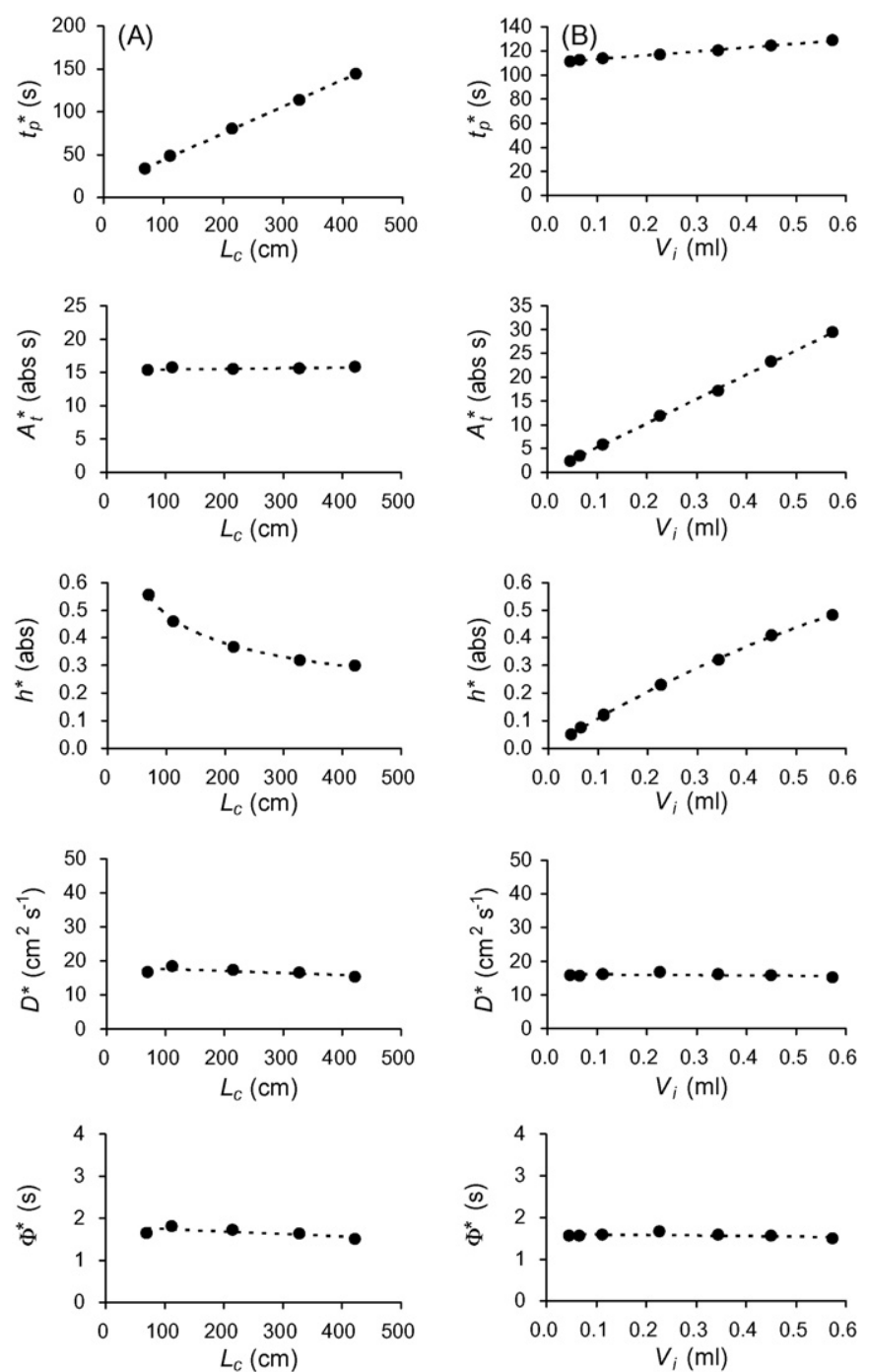

Fig. 4. Effects of changing (A) the coil length $L_{\mathrm{c}}$ and (B) the injection volume $V_{\mathrm{i}}$ to the peak parameters (i.e. peak position $t_{\mathrm{p}}^{*}$, peak area $A_{\mathrm{t}}^{*}$ and peak height $h^{*}$ ), empirical dispersion coefficient $D^{*}$ and temporal shift $\Phi^{*}$. The pumping rate for all tests was at $Q=0.016 \mathrm{ml} \mathrm{s}^{-1}$. Other conditions: $V_{\mathrm{i}}=0.112 \mathrm{ml}$ and $S_{0}=2.4$ for the experiment (A); $L_{\mathrm{c}}=328 \mathrm{~cm}$ and $S_{0}=0.89$ for the experiment (B).

lies between $+5 \%$ and $-3 \%$, but can exceed $\pm 10 \%$ when (i) the coil length is too short (i.e. $L_{\mathrm{c}}<112 \mathrm{~cm}$ ); (ii) the injection volume is too large (i.e. $V_{\mathrm{i}}>0.450 \mathrm{ml}$ ); and (iii) the pumping rate is too fast (i.e. $Q>0.208 \mathrm{ml} \mathrm{s}^{-1}$ ). Under these conditions, some physical mechanisms, classified as spatial asymmetrical factors, although minor, indeed exist in the flow system. Nonetheless, it shows that the temporal distortion effect is still the major factor controlling the peak-shape in flow injection analysis; further proofs since the first introduction of the concept several years ago [18].

\subsection{The "Nike" swoosh}

The interesting relationship between the pumping rate $Q$ and the apparent peak height $h^{*}$ (the "Nike" swoosh) was studied further. This phenomenon is quite similar to the two trends of dispersion variation that was described by $\mathrm{Li}$ and $\mathrm{Ma}$ [21]. It
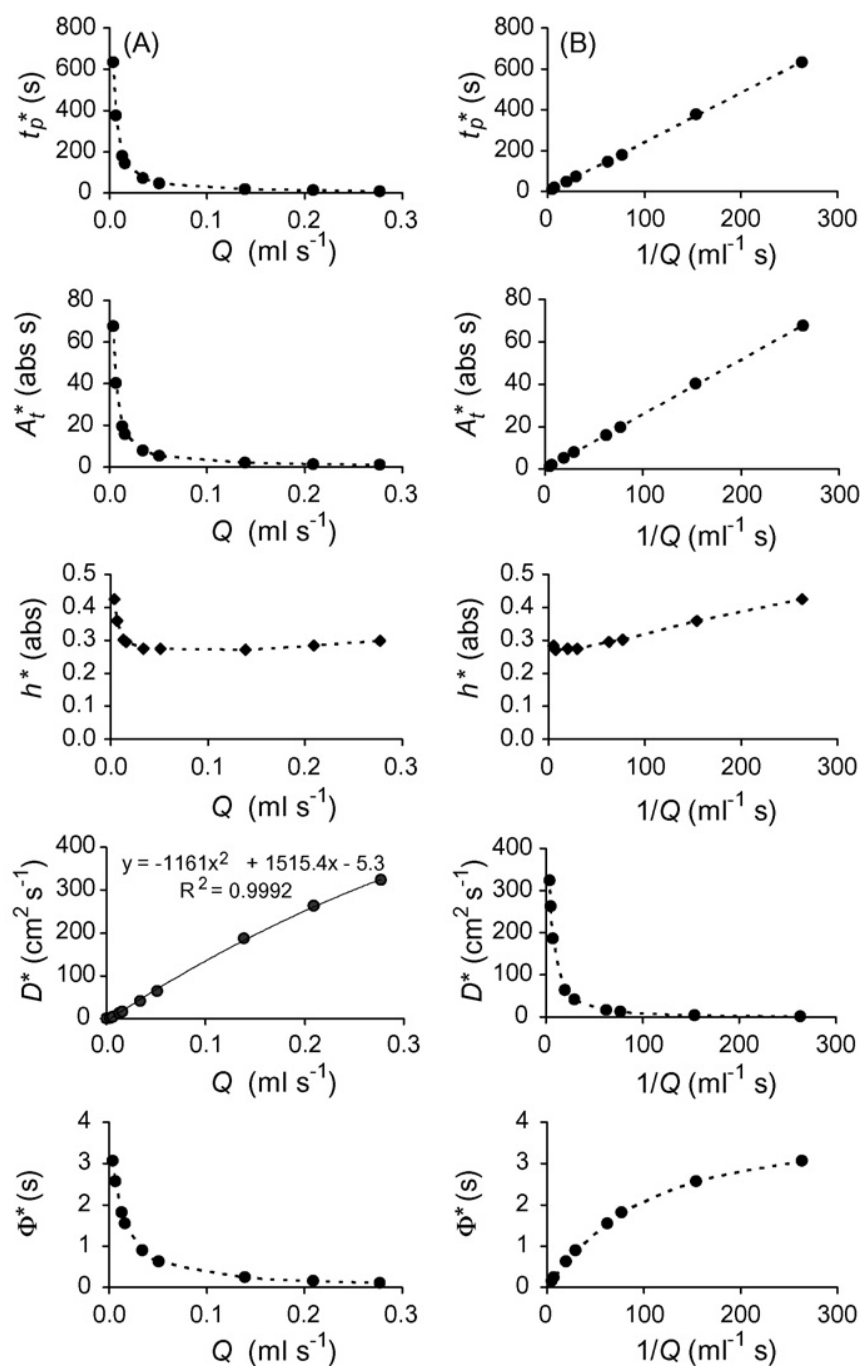

Fig. 5. Effect of the pumping rate to the peak parameters, dispersion coefficient and temporal shift. Data were plotted against (A) $Q$ and (B) $1 / Q$. The experimental conditions were: $L_{\mathrm{c}}=422 \mathrm{~cm}, V_{\mathrm{i}}=0.112 \mathrm{ml}$ and $S_{0}=2.49$.

can be seen that in Fig. 5(A), when $Q$ was decreased, the peak appearance time was delayed, the peak became wider but the peak height $\left(h^{*}\right)$ was "growing" higher. This last phenomenon is counter-intuitive that the peak height should be lower due to the longer residence time for dispersion. The only explanation would be that the dispersion is a function of the pumping rate $Q$, i.e. $D^{*}$ becomes smaller when $Q$ is slower.

Taking the data provided in Table 1, an empirical correlation was obtained by a fifth-order polynomial curve fitting (with no intercept):

$D^{*}(Q)=\delta_{1} Q+\delta_{2} Q^{2}+\delta_{3} Q^{3}+\delta_{4} Q^{4}+\delta_{5} Q^{5}$

where $\delta_{1}=729.3, \delta_{2}=17637, \delta_{3}=-164538, \delta_{4}=615341$ and $\delta_{5}=-832415$. However, when an intercept was allowed (ignoring the data at the slowest pumping rate), a much simpler second-order equation could be obtained:

$D^{*}(Q)=\delta_{0}+\delta_{1} Q+\delta_{2} Q^{2}$ 

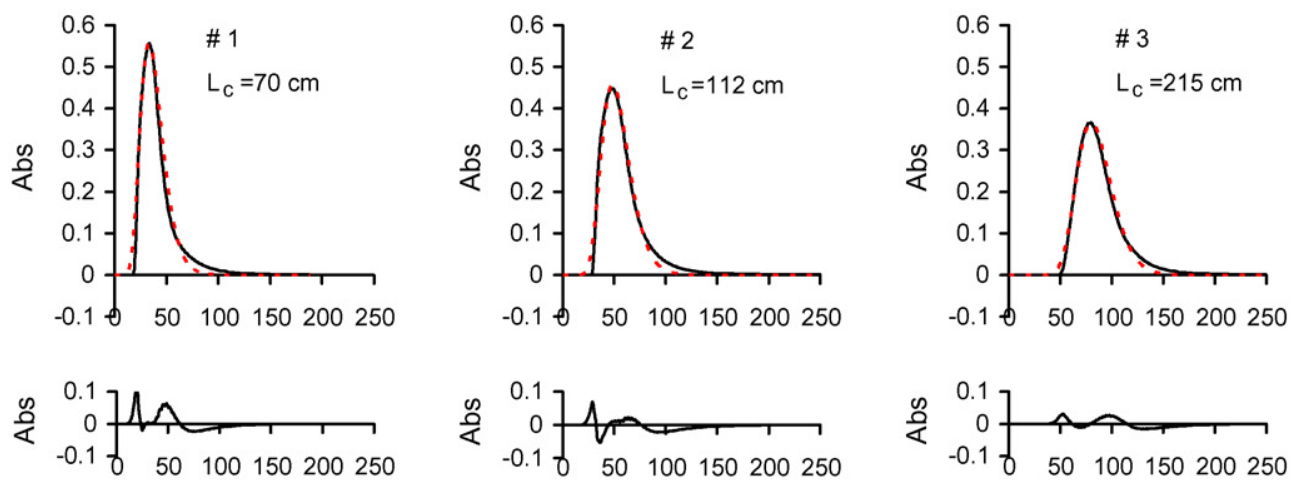

$\mathrm{t}(\mathrm{s})$
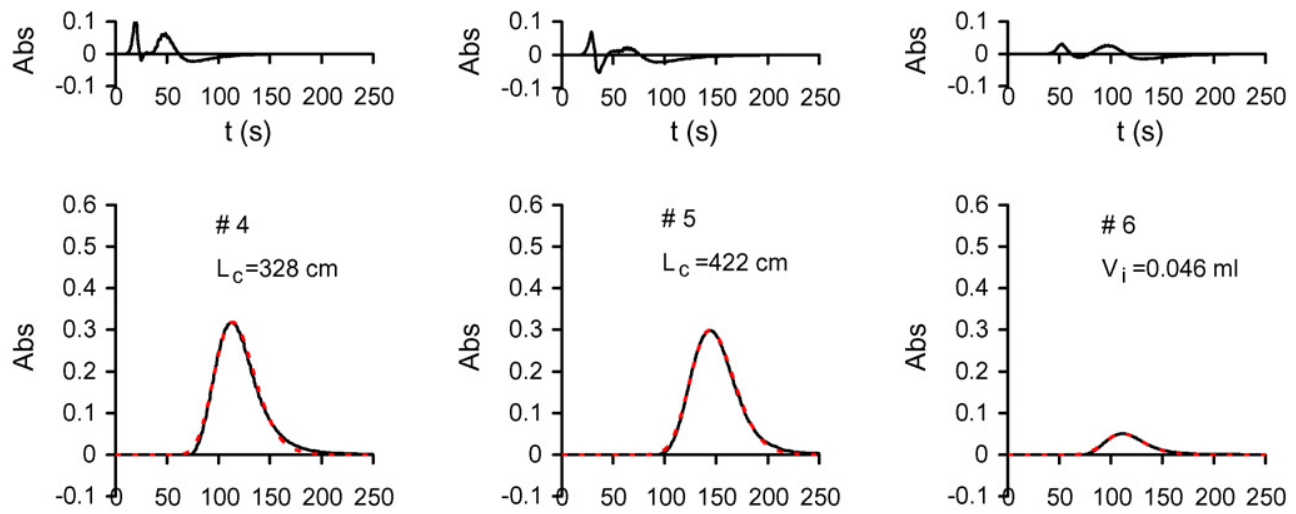

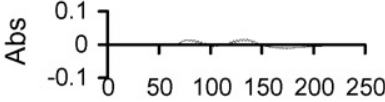

$\mathrm{t}$ (s)

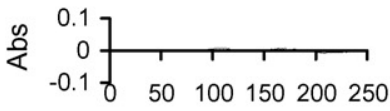

$t$ (s)
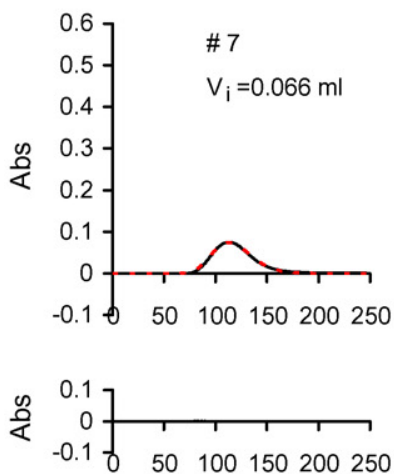

$\mathrm{t}$ (s)
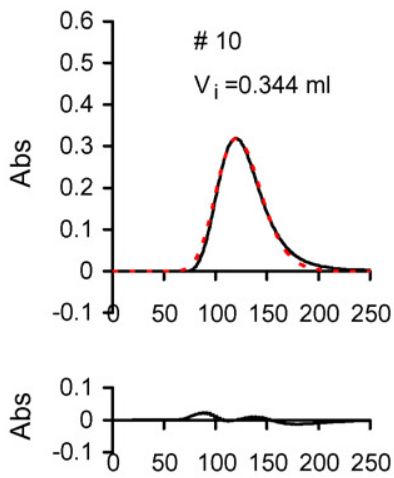

$\mathrm{t}(\mathrm{s})$
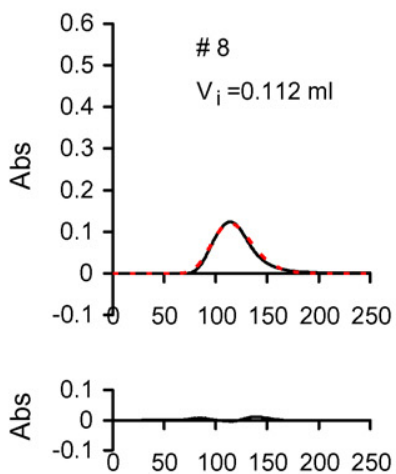

$\mathrm{t}(\mathrm{s})$
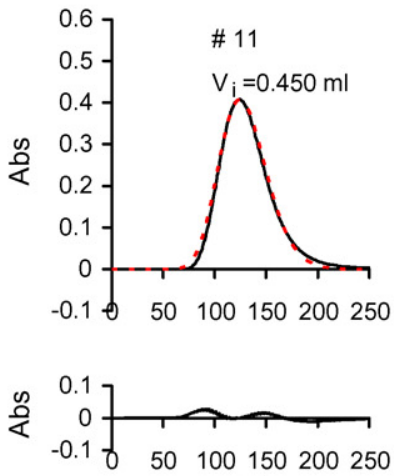

$\mathrm{t}(\mathrm{s})$

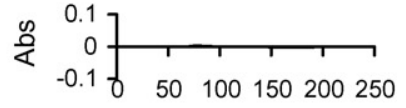

$\mathrm{t}$ (s)

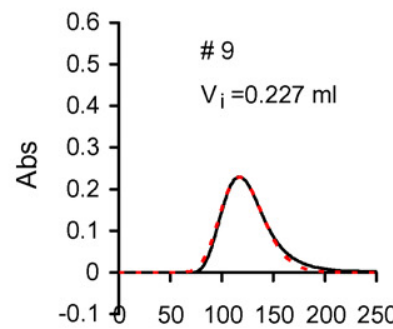

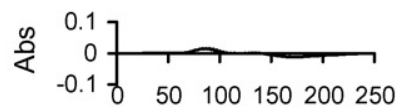

$\mathrm{t}$ (s)

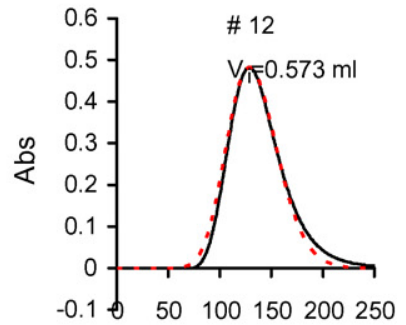

$\mathrm{t}$ (s)

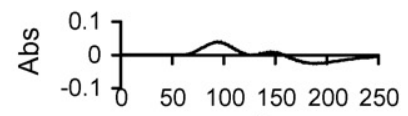

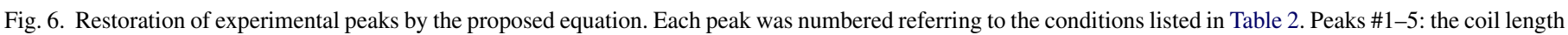

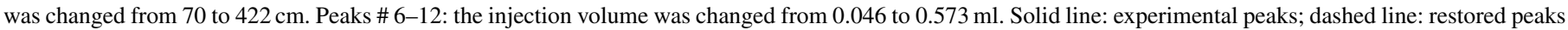
by Eq. (24). The residue curve is also plotted under each peak pair. 

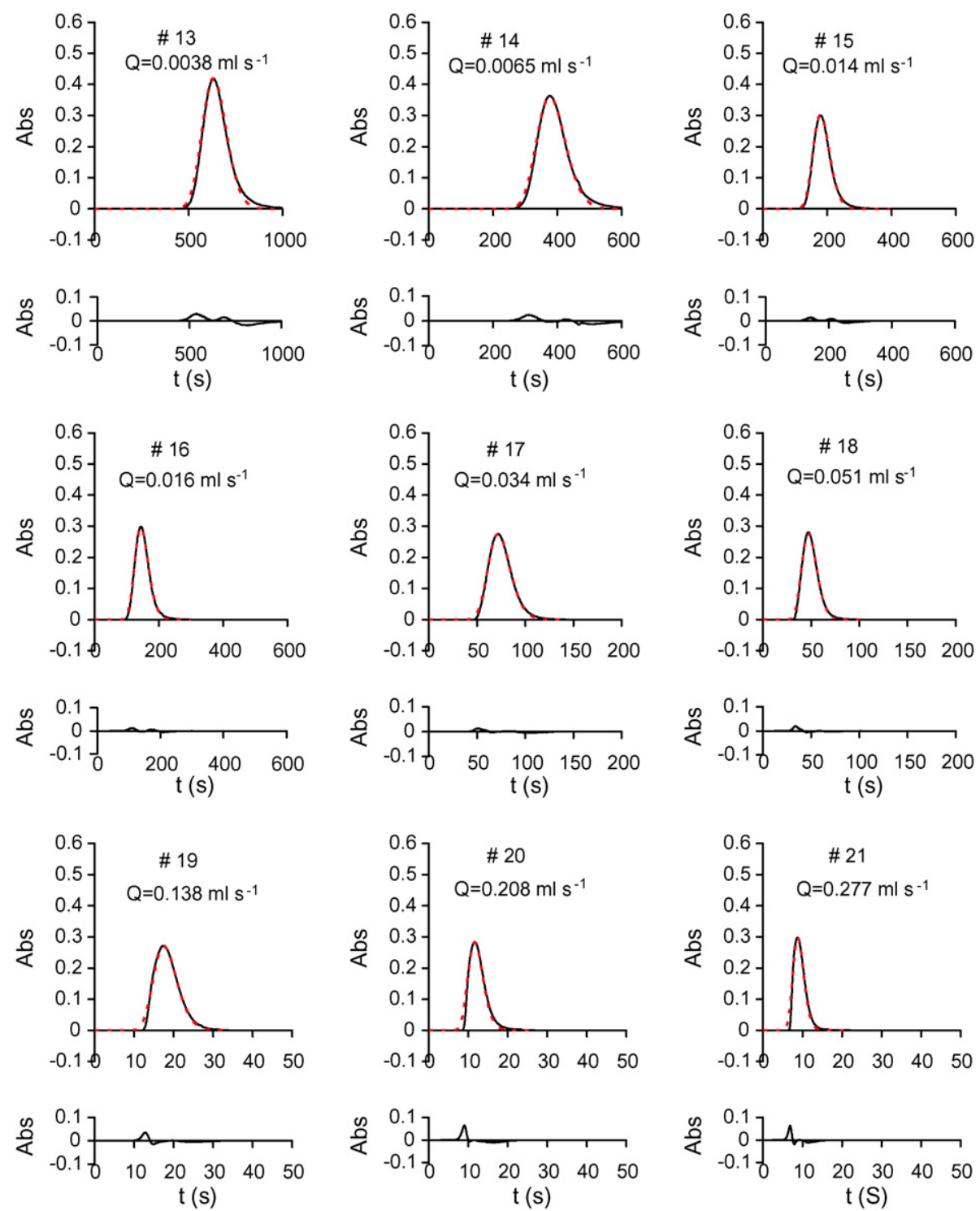

Fig. 7. Experimental and restored peaks for changing the pumping rate from 0.0038 to $0.277 \mathrm{ml} \mathrm{s}^{-1}$. Peak numbers (\#13-21) refer to experimental conditions listed in Table 2. Descriptions of peak curves are the same as Fig. 6.

where $\delta_{0}=-5.3, \delta_{1}=1515$ and $\delta_{2}=-1161$, with units of $\mathrm{cm}^{2} \mathrm{~s}^{-1}, \mathrm{~cm}^{-1}$ and $\mathrm{cm}^{-4} \mathrm{~s}$, respectively.

The use of the "negative" intercept $\left(\delta_{0}\right)$ reduces the complexity of the function from a 5 th-order to a 2nd-order, but it may be debatable even though it is just a mathematical fitting. If one enlarges the bottom part of the $D^{*}(Q)$ curve shown in Fig. 5, the $D^{*}$ value becomes relatively small when the pumping rate $Q$ is less than $0.0065 \mathrm{ml} \mathrm{s}^{-1}$. Additional tests using extremely slow pumping rates $\left(Q\right.$ between 0.0005 and $\left.0.003 \mathrm{ml} \mathrm{s}^{-1}\right)$ show that the $D^{*}$ value approaches gradually to zero (the sample plug takes more than $1000 \mathrm{~s}$ to arrive at the detector). Theoretically, when the flow is almost stationary, turbulence in the mixing coil is minimal, so the longitudinal dispersion is less impacted by the variation of the pumping rate. If the flow is completely stopped, then only the molecular diffusion is in charge, which should be a very small constant independent of the pumping rate.
Nonetheless, since the scale of the second term is dominating, it can be stated that the magnitude of dispersion in a flow injection system is basically proportional to the pumping rate within a limited working range (e.g. $Q$ between 0.0065 and $0.277 \mathrm{ml} \mathrm{s}^{-1}$ in this work).

Accordingly, the temporal shift also becomes a function of $Q$ :

$\Phi^{*}(Q)=-0.00013 Q^{-2}+0.03829 Q^{-1}-0.0293$

With Eqs. (27) and (28), one can generate peaks for a flow injection system at any given pumping rate within the valid range. Examples are given in Fig. 8. In this simulation, a sample plug $\left(V_{\mathrm{i}}=0.112 \mathrm{ml}\right)$ was injected into a flow system at pumping rates of $0.01,0.015,0.02,0.03,0.05,0.07,0.10,0.15,0.2$, 0.3 and $0.35 \mathrm{ml} \mathrm{s}^{-1}$, respectively. Three hypothetical detectors 

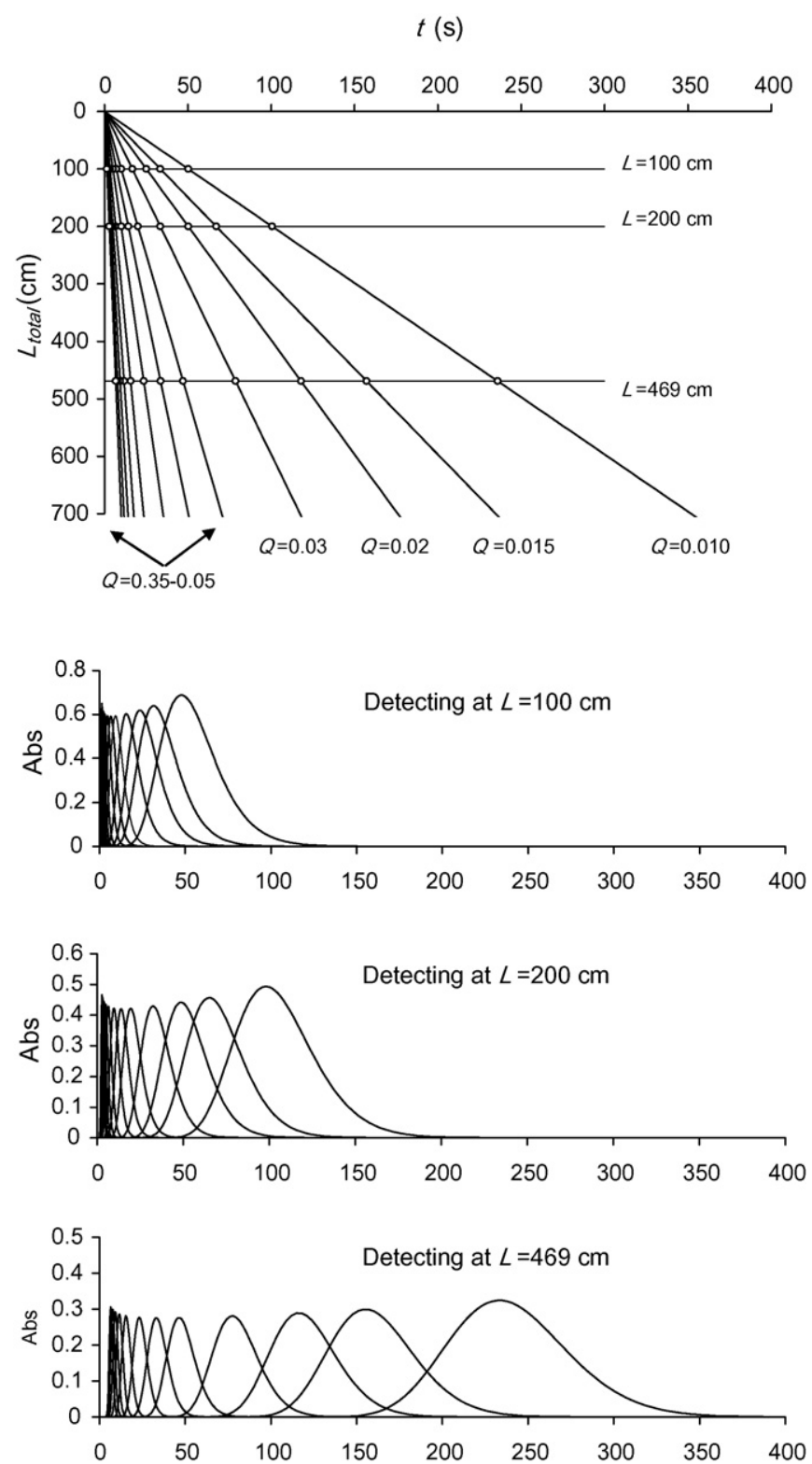

Fig. 8. Numerical simulations of the flow injection peaks at various pumping rates. A sample is injected into a flow system running at $Q=0.01,0.015,0.02$, $0.03,0.05,0.07,0.1,0.15,0.2,0.25,0.3$ and $0.35 \mathrm{ml} \mathrm{s}^{-1}$, respectively. Other conditions are $V_{\mathrm{i}}=0.112 \mathrm{ml}, S_{0}=2.49, \delta_{0}=-5.3, \delta_{1}=1515, \delta_{2}=-1161$. The migration routes are plotted on an $L$ vs. $t$ diagram, with three hypothetical detectors located at $L=100,200$ and $469 \mathrm{~cm}$, respectively. The peaks generated at all three positions show a "Nike" trend on peak height, while the peak areas are proportional to $1 / Q$.

were located at $L_{\text {total }}=100,200$ and $469 \mathrm{~cm}$. Other conditions were: $a=0.005027 \mathrm{~cm}^{2} ; S_{0}=2.49$. The peak shapes generated at each detecting position were plotted on an overlapped diagram (Fig. 8). It can be seen that the peak areas expand in proportion to $1 / Q$. The peak heights $\left(h^{*}\right)$ are relatively high at very fast pumping rates, but drop slightly as the pumping rate decreases, then raise when the pumping rate becomes very slow, an exact "Nike" trend like the experimental results shown in Fig. 5(A).

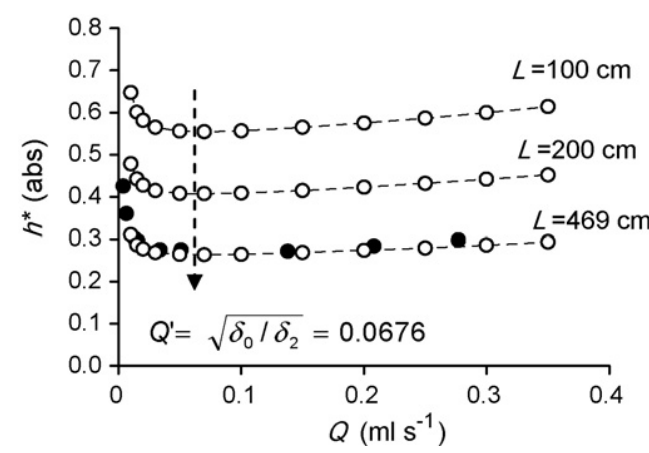

Fig. 9. The lowest point of the "Nike swoosh" is found at a pumping rate of $Q^{\prime}=\sqrt{\delta_{0} / \delta_{2}}$. Circles are simulated peak heights from Fig. 8 assuming that the detecting position is at $L=100,200$ and $469 \mathrm{~cm}$, respectively. Dots are experimental data for $L_{\text {total }}=469 \mathrm{~cm}$ taken from Table 2 .

The apparent peak height $h^{*}$ at a position $t_{\mathrm{p}}^{*}$ can be roughly estimated (ignoring the initial $\sigma_{\mathrm{L} 0}$ ) by:

$h^{*}\left(t_{\mathrm{p}}^{*}\right) \approx \frac{A_{\mathrm{t}}^{*}}{\sqrt{4 \pi D^{*} t_{\mathrm{p}}^{*} a^{2} / Q^{2}}}$

where all three variables (i.e. $t_{\mathrm{p}}^{*}, A_{\mathrm{t}}^{*}$ and $\left.D^{*}\right)$ are functions of $Q$. Among these, $A_{\mathrm{t}}^{*}(Q)=A_{\mathrm{L}} a / Q, t_{\mathrm{p}}^{*}(Q) \approx$ $L_{\mathrm{c}} a / Q$, and $D^{*}(Q)=\delta_{0}+\delta_{1} Q+\delta_{2} Q^{2}$. In combination, $h^{*} \propto$ $\sqrt{Q / D^{*}(Q)}$, or

$h^{*} \propto \sqrt{\frac{Q}{\delta_{0}+\delta_{1} Q+\delta_{2} Q^{2}}}$

This explains why the $h^{*}(Q)$ curve looks like a "Nike" shape, and why there exist two dispersion trends in a flow system. To locate the lowest point, a further differentiation of $h^{*}(Q)$ was made, which led to an equation:

$\delta_{0}-\delta_{2} Q^{2}=0$

Thus, the lowest peak height should occur at a pumping rate of

$Q^{\prime}=\sqrt{\delta_{0} / \delta_{2}}$

In the present case (see Fig. 9), $\delta_{0}=-5.3$ and $\delta_{2}=-1161$, therefore $Q^{\prime}=0.0676 \mathrm{ml} \mathrm{s}^{-1}$. In flow injection analysis, it is always expect that the sample zone should mix with reagents at the largest dispersion; therefore, the optimal pumping rate $Q_{\text {opt }}$ should be at $Q^{\prime}$. Up to this point, one may interestingly find that all the above descriptions for FIA are very similar to the search for an optimal flow rate for chromatography by the famous van Deemter equation.

\subsection{Throughput}

The three peak parameters $\left(h^{*}, t_{\mathrm{p}}^{*}\right.$ and $\left.A_{\mathrm{t}}^{*}\right)$ can also be used to give a measure of the analysis speed of a flow injection system with a fixed channel length operating at a given pumping rate. 
Since $h^{*}$ can be expressed as:

$h^{*} \approx \frac{A_{\mathrm{t}}^{*}}{\sqrt{2 \pi} \sigma_{\mathrm{t}}}$

where $\sigma_{\mathrm{t}}$ is the temporal standard deviation when the sample zone arrives at the detector at $t_{\mathrm{p}}^{*}$. The temporal width of a peak $W_{\mathrm{t}}$ is defined as $W_{\mathrm{t}}=4 \sigma_{\mathrm{t}}$, therefore,

$W_{\mathrm{t}}=\frac{4 A_{\mathrm{t}}^{*}}{\sqrt{2 \pi} h^{*}}$

By taking this width as a minimum time span to separate two sequentially injected samples, a maximum throughput $R$ (samples per hour) can be reasonably defined by:

$R\left(\mathrm{~h}^{-1}\right)=\frac{3600}{W_{\mathrm{t}}(s)}$

\section{Conclusion}

The present model has successfully demonstrated its usefulness in simulating peak shape, position and height for flow injection analysis. It is much simpler than most previous models, but the result matches all the characteristics that have been customarily used to describe the expanding nature of an injected sample plug in a tubular channel.

The model has several improvements over the previous work $[18,19]:$

(i) The sample size and the initial standard deviation have been included, which enables the modeling be feasible even when the sample size is not a small plug.

(ii) An observed dispersion coefficient $D^{*}$ has been defined, which can be reasonably estimated from the measurements of the experimental peak position, height and area.

(iii) An empirical temporal shift $\Phi^{*}$ can be calculated from $D^{*}$. It is nearly a constant for a given flow system operating at a fixed pumping rate. One can use it to modify the position term of a Gaussian function, so as to restore an experimental peak.

(iv) The relationship between $D^{*}$ and the pumping rate $Q$ has been related by an empirical function, i.e. $D^{*}(Q)=\delta_{0}+\delta_{1} Q+\delta_{2} Q^{2}$. The modeling of peak shapes can therefore be applied at different pumping rates.

(v) The optimal operation for a flow injection system can be find at a pumping rate of $Q_{\mathrm{opt}}=\sqrt{\delta_{0} / \delta_{2}}$.
However, small deviations on the peak shape due to physical effects cannot be denied. It could mean that the development of a sample band might not be completely symmetrical in the initial stage. If one insists, a further modification on the longitudinal standard deviation term (expressed as a polynomial function of time) [13-17] may be carried out. In this way, the spatial distribution pattern will become asymmetrical, and an even more skewed temporal peak image could be generated by a similar convolution process that has been described in this work.

\section{Acknowledgement}

The authors would like to thank S.C. Wong, H.T. Lin, C.S. Chern, G. Irby and an anonymous reviewer for their useful suggestions. This work is partially supported by the National Science Council, Taipei, Taiwan, under contract no. NSC942611-M-002-015.

\section{References}

[1] J. Ruzick, E.H. Hansen, Flow Injection Analysis, second ed., Wiley, New York, 1988

[2] S.D. Kolev, Anal. Chim. Acta 308 (1995) 36

[3] R. Tijssen, Anal. Chim. Acta 114 (1980) 71.

[4] S.D. Kolev, W.E. van der Linden, Anal. Chim. Acta 247 (1991) 51.

[5] D. Betteridge, C.Z. Marczewski, A.P. Wade, Anal. Chim. Acta 165 (1984) 227.

[6] P.D. Wentzell, M.R. Bowdridge, E.L. Taylor, C. MacDonald, Anal. Chim. Acta 278 (1993) 293.

[7] J.G. Atwood, M.J.E. Golay, J. Chromatogr. 218 (1981) 97.

[8] J.M. Reijn, W.E. van der Linden, H. Poppe, Anal. Chim. Acta 114 (1980) 105.

[9] J.M. Reijn, W.E. van der Linden, H. Poppe, Anal. Chim. Acta 126 (1981) 1 .

[10] S.D. Kolev, E. Pungor, Anal. Chem. 60 (1988) 1700

[11] J.P. Foley, J.G. Dorsey, Anal. Chem. 55 (1983) 730.

[12] J.P. Foley, J.G. Dorsey, J. Chromatogr. Sci. 22 (1984) 40.

[13] V.B. Di Marco, G.G. Bombi, J. Chromatogr. A 931 (2001) 1.

[14] J. Li, J. Chromatogr. A 952 (2002) 63.

[15] J.R. Torres-Lapasio, J.J. Baeza-Baeza, M.C. Garcia-Alvares-Coque, Anal. Chem. 69 (1997) 3822

[16] J.R. Torres-Lapasio, J.J. Baeza-Baeza, M.C. Garcia-Alvares-Coque, Anal Chem. 69 (1997) 3822

[17] R.D. Caballero, M.C. Gacia-Alvares-Cogue, J.J. Baeza-Baeza, J. Chromatogr. A 954 (2003) 59.

[18] S.C. Pai, J. Chromatogr. A 950 (2002) 271.

[19] S.C. Pai, L.Y. Chiao, J. Chromatogr. A, doi:10.1016/j.chroma.2006.11.012, in press.

[20] E.L. Cussler, Diffusion: Mass Transfer in Fluid Systems, Cambridge University Press, New York, 1997.

[21] Y. Li, H. Ma, Talanta 42 (1995) 2003. 